University of Nebraska - Lincoln

DigitalCommons@University of Nebraska - Lincoln

Faculty Publications, Department of Psychology

Psychology, Department of

$5-31-2019$

\title{
How self-compassion moderates the effect of body surveillance on subjective happiness and depression among women
}

\author{
Robin Wollast \\ Université libre de Bruxelles, Robin.wollast@ulb.ac.be \\ Abigail R. Riemer \\ University of Nebraska-Lincoln, ariemer@carrollu.edu \\ Philippe Bernard \\ Université libre de Bruxelles \\ Christophe Leys \\ Université libre de Bruxelles \\ Ilios Kotsou \\ Université libre de Bruxelles
}

See next page for additional authors

Follow this and additional works at: https://digitalcommons.unl.edu/psychfacpub

Part of the Psychology Commons

Wollast, Robin; Riemer, Abigail R.; Bernard, Philippe; Leys, Christophe; Kotsou, llios; and Klein, Olivier, "How self-compassion moderates the effect of body surveillance on subjective happiness and depression among women" (2019). Faculty Publications, Department of Psychology. 1061.

https://digitalcommons.unl.edu/psychfacpub/1061

This Article is brought to you for free and open access by the Psychology, Department of at DigitalCommons@University of Nebraska - Lincoln. It has been accepted for inclusion in Faculty Publications, Department of Psychology by an authorized administrator of DigitalCommons@University of Nebraska - Lincoln. 


\section{Authors}

Robin Wollast, Abigail R. Riemer, Philippe Bernard, Christophe Leys, Ilios Kotsou, and Olivier Klein 


\title{
How self-compassion moderates the effect of body surveillance on subjective happiness and depression among women
}

\author{
Robin Wollast, ${ }^{1}$ Abigail R. Riemer, ${ }^{2}$ Philippe Bernard, ${ }^{1}$ \\ Christophe Leys, ${ }^{1}$ Ilios Kotsou, ${ }^{1}$ and Olivier Klein ${ }^{1}$
}

1 Center for Social and Cultural Psychology, Université libre de Bruxelles, Bruxelles, Belgium

2 Department of Psychology, University of Nebraska-Lincoln, Lincoln, USA
Correspondence - Robin Wollast, Center for Social and Cultural Psychology, Department of Psychology, Université libre de Bruxelles, Avenue F. D. Roosevelt, 50, Campus du Solbosch, Brussels, Belgium. Email: Robin.Wollast@ulb.ac.be

ORCID: Robin Wollast https://orcid.org/oooo-0001-5395-9969

\begin{abstract}
According to objectification theory (Fredrickson \& Roberts, 1997), being treated as an object leads women to engage in self-objectification, which in turn increases body surveillance and body shame as well as impairs mental health. However, very little is known about what factors could act as buffers against the detrimental consequences of self-objectification. This paper seeks to understand the role of self-compassion (the ability to kindly accept oneself or show self-directed kindness while suffering) in the perception that women have of their own bodies. Results indicate that selfcompassion moderated the effect of body surveillance on depression and happiness separately among women. More specifically, for women low in self-compassion,
\end{abstract}

Published in Scandinavian Journal of Psychology 6o:5 (October 2019), pp 464-472.

DOI: $10.1111 /$ sjop.12553

Copyright (C) 2019 Scandinavian Psychological Associations and John Wiley \& Sons Ltd.

Used by permission.

Submitted 6 October 2018, accepted 16 April 2019; published 31 May 2019. 
body surveillance was negatively associated with happiness, which was explained by increased depression. In sum, our results indicate that self-compassion protects against the detrimental consequences of body surveillance.

Keywords: Body shame, body surveillance, happiness, psychological distress, selfcompassion, self-objectification.

If you talked to your friends the way you talk to your body, you'd have no friends left at all.

(Hutchinson, 1985, p. 14)

\section{Introduction}

Objectification theory developed by Fredrickson and Roberts (1997) postulates that Western societies sexually objectify women and consider them objects that can be looked at and judged by others. Twentytwo years later, sexual objectification - the tendency to consider or treat a person as a sexual object (Bartky, 1990) - is still present in various media (e.g., television, internet, music, magazines, video games) by conveying sexualized, idealized and stereotyped images of women (e.g., Flynn, Craig, Anderson \& Holody, 2016; Hatton \& Trautner, 2011; Wollast, Puvia, Bernard, Tevichapong \& Klein, 2018). Importantly, this sexualization in media outlets like Facebook (Fardouly, Diedrichs, Vartanian \& Halliwell, 2015), sexualizing sitcoms (Vandenbosch \& Eggermont, 2014), and sexualizing music videos (Karsay \& Matthes, 2016) increases women's exposure to sexualized ideals. Moreover, this pervasive exposure influences the way in which women perceive their own bodies (Vandenbosch \& Eggermont, 2012, 2013; Ward, Seabrook, Manago \& Reed, 2016), laying the groundwork for detrimental health behaviors (e.g., disordered eating behaviors, for a review see Moradi \& Huang, 2008).

A growing body of research has demonstrated the role of selfcompassion as a protective factor against poor body image and eating disorders (see Braun, Park \& Gorin, 2016 for a systematic review). Yet, "attempts to find variables that moderate the clinical symptomatology related to the objectification process have been largely unsuccessful" (Liss \& Erchull, 2015, p. 6). In fact, the few studies that focus on the relationship between constructs related to 
objectification theory (cf. objectified body consciousness) and selfcompassion have solely assessed mental health outcomes including body shame, eating disorders and depression (e.g., Daye, Webb \& Jafari, 2014; Liss \& Erchull, 2015).

This paper aims to overcome these limits. First, the current study aims to examine whether body surveillance predicts psychological distress and decreased subjective happiness. Second, we investigated whether women's self-compassion can be a protective factor in the relation between body surveillance and these negative outcomes. That is, we posited that the link between body surveillance and these detrimental consequences should be weaker among women high in selfcompassion than among women low in self-compassion.

\section{Sexual objectification}

Beginning in childhood, women are socialized to consider their bodies as objects rather than as active agents, and to view themselves from a third-person perspective (e.g., Fredrickson \& Roberts, 1997; Fredrickson, Roberts, Noll, Quinn \& Twenge, 1998). In this sense, being treated as an object leads women to engage in self-objectification focusing more on physical appearance rather than on competence. Because women live in a culture that primarily evaluates and appraises women based on their physical appearance, women who self-objectify might act as their own surveyor in order to gain power that is associated with fitting cultural beauty ideals (although this power is often restrictive given that women are conforming to patriarchal expectations, Fredrickson \& Roberts, 1997).

Importantly, the potential benefits of self-objectification are significantly outweighed by the mental health costs (see Moradi \& Huang, 2008, for a review; Szymanski, Moffitt \& Carr, 2011). For example, numerous investigations have demonstrated that self-objectification is associated with negative psychological consequences including anxiety about physical appearance and physical safety, low peak motivational states or flow, lower perception of internal body sensations, sexual dysfunction, depression, decreased well-being, and disordered eating behaviors (e.g., Fredrickson \& Harrison, 2005; Fredrickson \& Roberts, 1997; Gervais, Vescio, \& Allen, 2011; Miner-Rubino, Twenge \& Fredrickson, 2002). Additionally, failing to achieve a typically unattainable 
standard of beauty (e.g., a curvaceous yet thin body) typically results in body shame among women (e.g., McKinley \& Hyde, 1996; Noll \& Fredrickson, 1998). Importantly, self-objectification most commonly manifests in women's increased body surveillance; a plethora of studies have demonstrated that self-objectification is highly linked to body surveillance (e.g., McKinley \& Hyde, 1996; Morry \& Staska, 2001; Tylka \& Hill, 2004; Wollast, Coertjens, Bernard \& Klein, 2019).

Moreover, scholars have demonstrated that engaging in self objectification through body surveillance is associated with decreased well-being as indicated by higher reported depression (e.g., Muehlenkamp \& Saris-Baglama, 2002; Peat \& Muehlenkamp, 2011; Szymanski \& Henning, 2007) and decreased life satisfaction (e.g., Breines, Crocker \& Garcia, 2008; Mercurio \& Landry, 2008). Although life satisfaction is an important component of subjective well-being, life satisfaction is conceptually distinguishable from subjective happiness (Bartels \& Boomsma, 2009; Mattei \& Schaefer, 2004). According to Veenhoven (2010), happiness is one of the most fundamental human goals, which is associated with a large number of factors such as personality traits and life circumstances (e.g., socioeconomic level, family, marital status, Easterlin, 2003; Lyubomirsky, King \& Diener, 2005; Schimmack, 2008). Despite the importance of subjective happiness, very little is known regarding the relationships between self-objectification and subjective happiness. Recently, Swami, Tran, Stieger and Voracek (2015) revealed that body satisfaction positively predicts subjective happiness. With this link in mind, the present study aimed to examine the relationship between body surveillance, subjective happiness, and psychological distress (i.e., depression) thus, filling a gap in the current literature.

\section{Self-compassion}

Although the vast majority of studies have focused on the detrimental consequences of constructs posed by objectification theory, very little is known regarding the variables that can positively moderate the relationship between self-objectification and its negative consequences. Notably, a growing body of research (e.g., Albertson, Neff \& Dill-Shackleford, 2014; Liss \& Erchull, 2015) has suggested the importance of self-compassion - a factor that plays a critical role in the 
way that individuals perceive their own body - as a likely factor that could alleviate the association between self-objectification and its negative outcomes.

Self-compassion - the ability to kindly accept oneself or show self-directed kindness while suffering (Germer, 2009; Raes, 2011), comprises three interconnected components including: self-kindness, the perception of personal experience as common human experience, and mindfulness, each of which are positively associated with self-esteem (Neff, 2003). Research reveals that greater engagement in self-compassion positively impacts mental health outcomes including happiness, emotional intelligence, curiosity, wisdom, personal initiative, optimism and resilience (Heffernan, Quinn Griffin, McNulty \& Fitzpatrick, 2010; Hollis- Walker \& Colosimo, 2011; Leys, Arnal, Wollast, Rolin, Kotsou \& Fossion, 2018; Neff, Kirkpatrick \& Rude, 2007; Neff \& McGehee, 2010). Generally, self-compassion can be thought of as a buffer against negative feelings. Greater self-compassion effectively aids in coping with stressors such as academic pressure (Neely, Schallert, Mohammed, Roberts \& Chen, 2009), rumination and fear of failure (Neff, Hsieh \& Dejitterat, 2005), traumatic stress situations (Werner et al., 2012), and psychological vulnerability (Thompson \& Waltz, 2008). To illustrate, self-compassion moderates the link between affect and depression; although negative affect is related to depressive symptoms, being compassionate toward oneself reduces depression when expressing negative affect (Kotsou \& Leys, 2016). Moreover, self-compassion has a positive influence on psychological distress including: stress, depression, anxiety (e.g., Hall, Row, Wuensch \& Godley 2013; MacBeth \& Gumley, 2012; Neff \& McGehee, 2010; Olson, Kemper \& Mahan, 2015), social anxiety (Gilbert \& Procter, 2006) and feelings of shame (Ferreira, Pinto-Gouveia \& Duarte, 2013).

\section{The role of self-compassion in body image}

Alongside the literature investigating the influence of self-compassion on mental health outcomes, a growing body of research has examined the links between self-compassion and body image, including constructs related to objectification theory (see Braun et al., 2016 for a review). The three interconnected components of self-compassion 
positively influence the relationship between body image and self-esteem (Neff, 2003). The relationship between body image satisfaction and self-esteem becomes less stringent for women with greater levels of self-compassion (Pisitsungkagarn, Taephant \& Attasaranya, 2014). Importantly, self-compassion is negatively associated with body surveillance and body shame - two primary manifestations of self-objectification (Daye et al., 2014; Mosewich, Kowalski, Sabiston, Sedgwick \& Tracy, 2011). Moreover, self-compassion partially mediates the relationship between two psychological outcomes of self-objectification - body dissatisfaction and depression (Wasylkiw, MacKinnon \& MacLellan, 2012). Additionally, research reveals body surveillance, body shame, depression, and negative eating attitudes are lower for women with greater self-compassion (Liss \& Erchull, 2015). In the same vein, while self-compassion has been associated negatively with unhealthy eating concerns (e.g., Kelly, Carter \& Borairi, 2014; Prowse, Bore \& Dyer, 2013; Webb \& Forman, 2013), high levels of self-compassion are positively associated with healthy motivation for exercise (e.g., Magnus, Kowalski \& McHugh, 2010).

Relatedly, it is well established that self-compassion interventions have positive effects on body image and contribute to decreased selfobjectification (see Rahimi-Ardabili, Reynolds, Vartanian, McLeod \& Zwar, 2017 for a systematic review). For example, Albertson et al. (2014) tested the effects of self-compassion on body satisfaction among adult women by using a brief 3-week period of meditation training. Each week, women who were randomized to the intervention group received a link to a podcast containing 20-minute self-compassion meditation sessions (Mindful Self-Compassion program, Neff \& Germer, 2013). As a result of the self-compassion meditation, relative to the control group, women in the intervention group experienced reduced manifestations of self-objectification: reporting less body dissatisfaction, less body shame, and less contingent self-worth based on appearance, in addition to increased body appreciation, immediately after the intervention and 3 months later. Recent conceptual replications have demonstrated similar self-compassion intervention results on body surveillance by using yoga-based physical education (Cox, Ullrich-French, Cole \& D'Hondt-Taylor, 2016; Cox, Ullrich-French, Howe \& Cole, 2017). 


\section{The present study}

The scientific and societal challenge of this paper is to understand the role of self-compassion in women's perceptions of their bodies. Specifically, we suggest that self-compassion may protect against the detrimental consequences (psychological distress and body shame) of body surveillance (accepting imperfections rather than self-criticism and judgements) and improve subjective happiness.

\section{Hypotheses and overview of current work}

In the current work, we proposed that body surveillance would predict body shame positively (H1a), psychological distress positively (H1b), and happiness negatively (H1c). Second, we expected that selfcompassion would predict body shame negatively (H2a), psychological distress negatively ( $\mathrm{H}_{2} \mathrm{~b}$ ), and happiness positively ( $\mathrm{H} 2 \mathrm{c}$ ). Third, we postulated that self-compassion would moderate the relations between body surveillance and body shame (H3a), body surveillance and psychological distress (H3b), as well as body surveillance and happiness (H3c); with self-compassion lessening the impact of body surveillance on each outcome. Finally, we hypothesized a mediated moderation in which depression would mediate the effect of self-compassion on the relation between body surveillance and happiness. In particular, depression was hypothesized to mediate the relation between body surveillance and happiness, and self-compassion was expected to moderate pathways between body surveillance and depression and body surveillance and happiness ( $\left.\mathrm{H}_{4}\right)$.

\section{Method}

\section{Participants}

Relying on similar previous work on self-compassion and body image (e.g., Kelly, Vimalakanthan \& Miller, 2014; Pisitsungkagarn et al., 2014) the sample size for the current study was calculated assuming a moderate effect size $\left(f^{2}=0.15\right)$ with a power of 0.95 , which translated into a sample size of 119. In total, 135 Belgian women with French 
as their native language participated in this study. Participants' age ranged from 18 to 69 years $(M=24.99 ; S D=10.71)$. Participants were predominantly first-year students $(>90 \%)$, and there was little variation in participants' reported sexual orientation ( $91 \%$ heterosexual, $5 \%$ bisexual, $3 \%$ lesbian, and $1 \%$ other) and marital status (56\% single, $35 \%$ in a committed relationship but not married, $7 \%$ married, $1 \%$ divorced, and $1 \%$ widowed). Participants' cities of residence were mostly urban industrialized sites (e.g., Brussels). The mean calculated BMI score fell within the normal weight range $(M=22.07, S D=3.22)$. Participants' BMIs reflected the following classifications: $11 \%$ underweight, $74 \%$ normal weight, $12 \%$ over-weight, and $3 \%$ obese.

Participants were mostly recruited in a Belgian (French speaking) University and completed the questionnaire either voluntarily or for course credit. Specifically, the vast majority of participants (>90\%) were asked to fill in an online questionnaire about "women's body image" for a psychology class. Importantly, participants have not been previously involved in the study, and had no prior knowledge of the objectives or its specific context. Furthermore, they were ensured that their responses would remain anonymous and kept confidential. At the end of the questionnaire, participants were thanked and fully debriefed. We also posted the online survey on students' work group webpages associated with their university to collect more participants.

\section{Measures}

Participants completed a single online questionnaire. All instruments administered were translated in French following the guidelines given by Brislin and colleagues (Brislin, 1970; Wallace \& Brislin, 1973). The systematic approach to translation was selected for its assurance of construct equivalence rather than verbatim equivalence between the original and target languages. Within the questionnaire, participants self-reported: psychological distress, subjective happiness, body surveillance, body shame, and self-compassion. After completing the survey, participants provided socio-demographic information. All dependent variables were presented in the same order but items within the scales were presented in a randomized order to reduce common method variance problems (Podsakoff, MacKenzie, Lee \& Podsakoff, 2003). 
Psychological distress. To assess psychological distress, we used the 21- item Depression, Anxiety and Stress Scale (DASS21; Lovibond \& Lovibond, 1995; Crawford \& Henry, 2003). Specifically, we used the French validated version of the DASS21 (EDAS; Ramasawmy, 2015). The DASS21 comprises three subscales measuring depression (e.g., "I couldn't seem to experience any positive feeling at all," "I felt that I had nothing to look forward to"), anxiety (e.g., "I was worried about situations in which I might panic and make a fool of myself," "I felt scared without any good reason"), and stress (e.g., "I found it hard to wind down," "I found it difficult to relax"). Participants ranked each statement on a four-point Likert scale ranging from o (did not apply to me at all) to 3 (applied to me very much or most of the time). Responses were averaged so that higher scores indicate greater psychological distress. All subscales were associated with good internal consistencies ( $\alpha=0.80$ for Depression; $\alpha=0.76$ for Anxiety; $\alpha=0.82$ for Stress).

Subjective happiness. To assess subjective happiness, we used the four item Subjective Happiness Scale (SHS; Lyubomirsky \& Lepper, 1999). Specifically, we used the French translated and validated version of the SHS (Kotsou \& Leys, 2017). Participants completed four statements (e.g., "In general I consider myself") using a seven-point Likert scale ( $1=$ Not a very happy person, $7=A$ very happy person). Mean scores were calculated so that higher scores reflect greater happiness $(\mathrm{a}=0.87)$.

Body surveillance. To measure the extent to which participants monitor their appearance, we used a dimension of the Objectified Body Consciousness Scale (OBCS, McKinley \& Hyde, 1996). Specifically, we asked participants to complete the Body Surveillance Subscale in which they were asked to rate their agreement with eight items (e.g., "During the day, I think about how I look many times.," "I often worry about whether the clothes I am wearing make me look good.") using a 1 (strongly disagree) to 7 (strongly agree) Likert scale. Higher scores indicate more body surveillance. Similar to the original English version, internal consistency was acceptable $(\alpha=0.75)$. 
Body shame. Participants' feelings of body shame were assessed using the Body Shame Subscale of the OBCS (McKinley et al., 1996). Participants were asked to rate their agreement with eight items (e.g., "I would be ashamed for people to know what I really weigh." "I feel ashamed of myself when I haven't made the effort to look my best.”) using a 1 (strongly disagree) to 7 (strongly agree) Likert scale. Higher scores indicate greater feelings of body shame. Like the English version, internal consistency was good ( $\alpha=0.85)$.

Self-compassion. To measure self-compassion, participants completed the Self-Compassion Scale (SCS, Raes, Pommier, Neff \& Van Gucht, 2011). Specifically, we used the French translated and validated version of the SCS (EAC; Kotsou \& Leys, 2016). This scale contains 26 statements that can be divided in six sub-scales: selfkindness (e.g., "I try to be understanding and patient towards those aspects of my personality I don't like.”), self-judgment (e.g., "I'm disapproving and judgmental about my own flaws and inadequacies."), common humanity (e.g., "When I feel inadequate in some way, I try to remind myself that feelings of inadequacy are shared by most people."), isolation (e.g., "When I fail at something that's important to me, I tend to feel alone in my failure.”), mindfulness (e.g., "When something painful happens I try to take a balanced view of the situation"), and over-identification (e.g., "When I fail at something important to me I become consumed by feelings of inadequacy."). Items are rated on a 5-point response scale ranging from 1 (almost never) to 5 (almost always). Item responses were reversed where necessary and averaged to create a single self-compassion score with higher scores reflecting ability to be kind to oneself, to recognize that one's experience is a part of the shared human condition, and to be able to not over-identify with strong negative emotions and self-evaluative thoughts $(\alpha=0.90)$.

\section{Results}

The means, standard deviations and correlations of all variables are reported in Table 1. In line with hypotheses, body surveillance is correlated positively with body shame (Hypothesis 1a), and negatively with happiness (Hypothesis 1c). Contradictory to Hypothesis 
Table 1. Corresponding means and correlation matrix of all variables

\begin{tabular}{|c|c|c|c|c|c|c|c|c|}
\hline & Mean (SD) & Body shame & Self-compassion & Happiness & Depression & Anxiety & Stress & $B M I$ \\
\hline Body surveillance & $4.79(1.03)$ & $0.45^{* * *}$ & $-0.33^{* * *}$ & $-0.24^{* *}$ & $0.26 * *$ & 0.14 & 0.11 & -0.08 \\
\hline Body shame & $3.02(1.4)$ & - & $-0.47^{* * *}$ & $-0.23^{* *}$ & $0.35^{* * *}$ & $0.25^{* *}$ & $0.27^{* *}$ & $0.32^{* * *}$ \\
\hline Self-compassion & $2.63(0.65)$ & - & - & $0.53^{* * *}$ & $-0.47^{* * *}$ & $-0.42^{* * *}$ & $-0.37^{* * *}$ & 0.09 \\
\hline Happiness & $4.68(1.29)$ & - & - & - & $-0.51^{* * *}$ & $-0.34^{* * *}$ & $-0.31^{* * *}$ & 0.09 \\
\hline Depression & $0.86(0.54)$ & - & - & - & - & $0.61^{* * *}$ & $0.67^{* * *}$ & 0.02 \\
\hline Anxiety & $0.65(0.55)$ & - & - & - & - & - & $0.64^{* * *}$ & -0.10 \\
\hline Stress & $1.09(0.59)$ & - & - & - & - & - & - & 0.02 \\
\hline BMI & $22.07(3.22)$ & - & - & - & - & - & - & - \\
\hline
\end{tabular}

**Correlation is significant at the 0.01 level.

$* * *$ Correlation is significant at the 0.001 level.

$1 \mathrm{~b}$, body surveillance did not correlate with the overarching concept of psychological distress. Yet, because of our interest in psychological outcomes we further explored the relation between body surveillance and the components of psychological distress. In partial support for Hypothesis $1 \mathrm{~b}$, body surveillance is positively correlated with one of the components of psychological distress - depression, but not anxiety and stress. As additional support for our hypotheses, self-compassion is negatively correlated with body shame (Hypothesis 2a), negatively correlated with each of the components of psychological distress - depression, anxiety, and stress (Hypothesis $2 \mathrm{~b}$ ), and positively correlated with happiness (Hypothesis 2c).

A series of regression analyses was also used to test the hypotheses. Unless otherwise indicated, we relied on the same analysis strategy using mean-centered variables. Specifically, body surveillance was included as the predictor, self-compassion as the moderator, and body shame, psychological distress and happiness were included as outcome variables. BMI and age were entered as covariates. Controlling for body mass index and age did not alter the results, and we thus excluded them.

\section{Body shame}

First, we implemented a multiple linear regression with body surveillance and self-compassion as predictors. At step 1, results showed that body surveillance positively predicted body shame ( $\beta=0.46, p$ $<$ o.001, H1a), and self-compassion negatively predicted body shame 
$(\beta=-0.77, p<0.001, \mathrm{H} 2 \mathrm{a})$. In a second step, we entered the interaction between the two predictors. Contrary to Hypothesis 3a, the Body Surveillance $\times$ Self-Compassion interaction was not significant $(\beta=-0.13, p=0.394)$. While these results suggest that self-compassion does not moderate the link between body surveillance and body shame among Belgian women, there is the possibility that our sample size was too small to find this effect. We return to this possibility within the discussion.

\section{Depression, anxiety and stress}

Using the same strategy for depression, we found that, at step 1, body surveillance did not predict depression $(\beta=0.06, p=0.016, \mathrm{H} 1 \mathrm{~b})$ but self-compassion negatively predicted depression $(\beta=-0.36, p<$ 0.001, H2b). Most importantly, in line with Hypothesis 3b, the Body Surveillance $\times$ Self-Compassion interaction, entered at step 2, was significant $\left(\beta=-0.14, p=0.023, \Delta \mathrm{R}^{2}=0.03\right)$, suggesting that self-compassion negatively moderated the link between body surveillance and depression among Belgian women. For women who have high levels of self-compassion, their depression levels remained low regardless of their engagement in more or less body surveillance. Whereas women low in self-compassion experienced more depression than women high in self-compassion, and depression was heightened when engaging in more relative to less body surveillance (see Figure 1). Note that, in line with Hypothesis $2 \mathrm{~b}$, self-compassion predicted anxiety $(\mathrm{b}=-0.35, p$ $<0.001)$, and stress ( $\beta=-0.34, p<0.001)$ negatively. However, contrary to Hypotheses $1 \mathrm{~b}$ and $3 \mathrm{~b}$, the main effects of body surveillance at step $1\left(\beta_{\text {anxiety }}=0, p=0.973 ; \beta_{\text {stress }}=-0.01, p=0.857\right)$ and the interactions $\left(\beta_{\text {anxiety }}=-0.01, p=0.834 ; \beta_{\text {stress }}=-0.09, p=0.231\right)$ between both predictors at step 2 were not significant for anxiety and stress.

\section{Happiness}

When performing the same analysis with happiness as the dependent variable, multiple regressions revealed that, in step 1 , body surveillance did not predict happiness $(\beta=-0.1, p=0.308, \mathrm{H} 1 \mathrm{c})$, but selfcompassion positively predicted happiness ( $\beta=0.99, p<0.001, \mathrm{H} 2 \mathrm{c}$ ). Most importantly, in line with Hypothesis 3c, the Body Surveillance $\times$ Self-Compassion interaction entered at step 2 was significant $(\beta=$ 


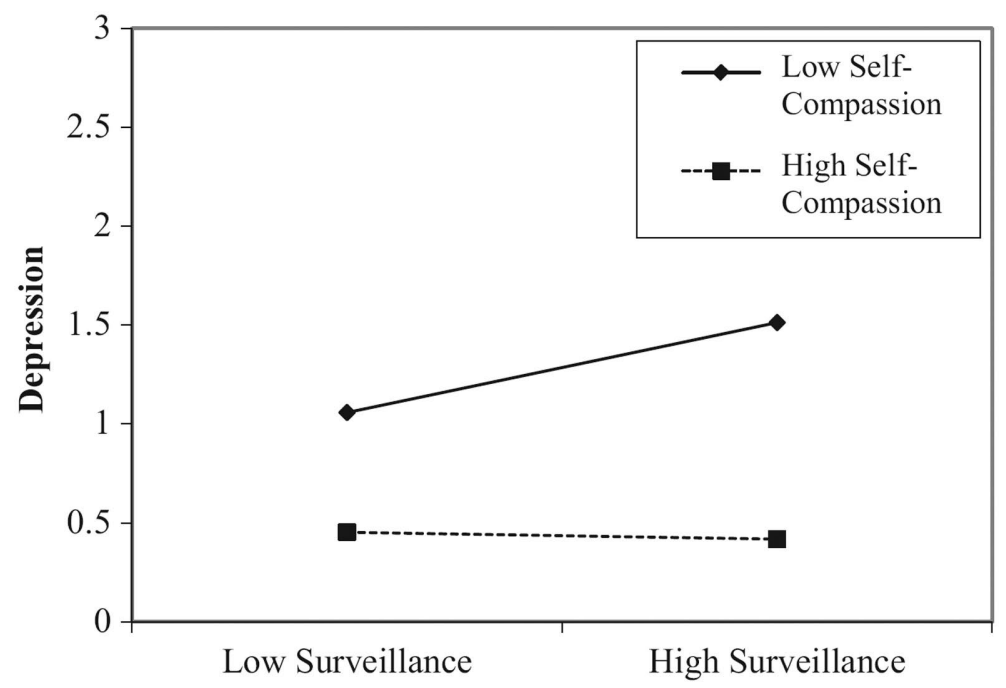

Figure 1. Depression as predicted by body surveillance with low and high self-compassion among Belgian women.

0.29, $\left.p=0.041, \Delta R^{2}=0.03\right)$, suggesting that self-compassion positively moderated the link between body surveillance and happiness. As expected, women high in self-compassion reported greater happiness than women low in self-compassion regardless of whether they were engaging in more or less body surveillance. Yet, for women low in self-compassion engaging in more body surveillance led to less happiness than engaging in less body surveillance (see Figure 2).

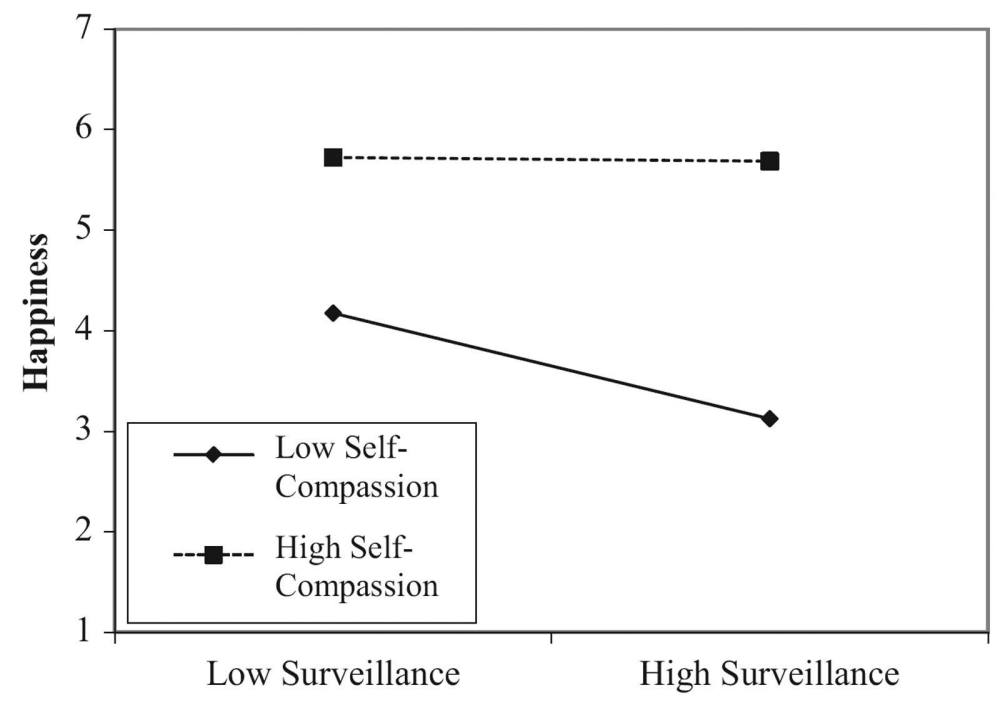

Figure 2. Happiness as predicted by body surveillance with low and high selfcompassion among Belgian women. 


\section{Sociodemographic characteristics}

For exploratory purposes, we tested if differences exist between categories of BMI (underweight, normal weight, over-weight, and obese) sexual orientation (heterosexual, bisexual and lesbian) and marital status (single, in a committed relationship but not married, married, divorced and widowed) on the dependent variables. The results of the ANOVA indicated non-significant effects of BMI, marital status and sexual orientation on all dependent variables $(p>0.05$ for each). Importantly, these results should be carefully interpreted given the underrepresentation of participants in some categories.

\section{Mediated moderation}

Further, the fourth hypothesis required a mediated moderation model (Muller, Judd \& Yzerbyt, 2005) to be tested. In this case, body surveillance is considered to be a predictor (X), self-compassion is the moderator (Mo), depression is the mediator (Me), and happiness is the outcome variable (Y). BMI and age were entered as covariates. Controlling for body mass index and age did not alter the results, and therefore we excluded them as covariates.

Showing that the effect of body surveillance on happiness is moderated by self-compassion and that this moderation is mediated by depression requires three steps (see Table 2). The first step (equation 1) reproduces the result of the preceding linear regression analysis and shows a significant interaction, predictor (body surveillance)

Table 2. Mediated moderation

\begin{tabular}{llll} 
& $\begin{array}{l}\mathrm{X}+\mathrm{Mo}+\mathrm{X} \times \mathrm{Mo}=\mathrm{Y} \\
\text { Equation 1 }\end{array}$ & $\begin{array}{l}\mathrm{X}+\mathrm{Mo}+\mathrm{X} \times \mathrm{Mo}=\mathrm{Me} \\
\text { Equation 2 }\end{array}$ & $\begin{array}{l}\mathrm{X}+\mathrm{Mo}+\mathrm{X} \times \mathrm{Mo}+\mathrm{Me}+\mathrm{Me} \times \mathrm{Mo}=\mathrm{Y} \\
\text { Equation 3 }\end{array}$ \\
\hline Predictor $(\mathrm{X})$ & $\beta_{11}=-0.1$ & $\beta_{21}=0.06$ & $\beta_{31}=-0.06$ \\
Moderator $(\mathrm{Mo})$ & $\beta_{12}=0.96^{* * *}$ & $\beta_{22}=-0.35^{* * *}$ & $\begin{array}{l}\beta_{32}=0.68^{* * *} \\
\mathrm{X} \times \text { Mo }\end{array}$ \\
$\beta_{13}=0.29^{*}$ & $\beta_{23}=-0.14^{*}$ & $\beta_{33}=0.21$ \\
Mediator $(\mathrm{Me})$ & - & - & $\beta_{34}=-0.79^{* * *}$ \\
Me $\times$ Mo & - & - & $\beta_{35}=-0.17$
\end{tabular}

$\mathrm{X}=$ Body surveillance; $\mathrm{Mo}=$ Self-compassion; $\mathrm{Y}=$ Happiness; $\mathrm{Me}=$ Depression.

*Correlation is significant at the 0.05 level.

$* * *$ Correlation is significant at the 0.001 level. 
$\times$ moderator (self-compassion), on the outcome variable (happiness), this model account for $30 \%$ of the variance $\left(R^{2}=0.304\right), F(3,131)=$ 19.067 , and $p<0.001$. The second step (equation 2) reproduces the result of the preceding linear regression analysis and shows a significant interaction (predictor $\times$ moderator) on the mediator (depression), this model account for $26 \%$ of the variance $\left(R^{2}=0.264\right), F(3$, $131)=15.646$, and $p<0.001$. The third step considers the effect of five IVs on the outcome variable (equation 3): (1) Predictor; (2) moderator; (3) predictor 9 moderator; (4) mediator; (5) mediator $\times$ moderator.

For a moderation to be mediated, there must be a significant interaction (predictor $\times$ moderator) in step 1 (equation 1 ) that becomes non-significant in step 3 (equation 3 ). This loss of significance is equal to the sum of two products: $\beta_{13}-\beta_{33}=\beta_{21} \beta_{35}+\beta_{23} \beta_{34}$ (see Table 2). One of the two products of the left member must be significant. As observed in Table 2, depression mediates the link between body surveillance and happiness, $F(5,129)=15.76$, and $p<0.001$. This model accounts for $38 \%$ of the variance $\left(R^{2}=0.379\right)$. The interaction between the predictor and moderator is significant in equation 1 and becomes non-significant in equation 3 , whereas the predictor $\left(\beta_{23}=-0.14^{*}\right)$ has a significant effect on the mediator, and the mediator $\left(\beta_{34}=-0.79^{* * *}\right)$ has a significant effect on the outcome variable. Therefore, the significant indirect effect is $(-0.14)(-0.79)=0.11$. Together these results indicate that the mediation is complete and suggest that the relation between body surveillance and happiness is mediated by depression and that the relations between body surveillance and both outcomes is moderated by self-compassion.

\section{Discussion}

Based on previous findings, the present study furthers our understanding of the consequences of women's body surveillance and offers evidence regarding the positive effect of self-compassion on the way women perceive their own body. Indeed, our results demonstrated that being compassionate with oneself attenuates the harmful consequences of body surveillance with beneficial effects among women. Self-compassion moderated the effect of body surveillance on depression and happiness, independently, among women. More specifically, 
we found that for women low in self-compassion, body surveillance is negatively associated with happiness, which is explained by increased depression among these women. However, even if self-compassion negatively predicted body shame, it is not strong enough for women to alleviate from this primary negative consequence of body surveillance.

\section{Self-compassion, body image and mental health}

First and as expected, we found that self-compassion was negatively associated with body surveillance, body shame, depression, anxiety and stress, and positively associated with happiness. This study replicates well-established findings in the literature suggesting that self-compassion has a positive influence on mental health (e.g., Albertson et al., 2014; Heffernan et al., 2010; Hollis-Walker \& Colosimo, 2011; Liss \& Erchull, 2015; Neff et al., 2007). Second, we also found that body shame was strongly and positively associated with body surveillance and variables indicating poor mental health. These results are consistent with previous studies revealing that body shame impairs mental health (cf. Moradi \& Huang, 2008; Szymanski et al., 2011). Moreover, we found that body surveillance was associated with more depression and less happiness, but it did not predict stress or anxiety. Thus, body surveillance could be seen as a trait that coincides with self-objectification, commonly leading women to engage in comparison with others (e.g., beauty standards, friends, strangers), based on appearance, while body shame can be seen as a negative consequence of this comparison; most likely to occur because of extreme expectations regarding beauty standards (cf. McKinley \& Hyde, 1996).

Interestingly, some women believe that they generally gain power through their appearance and especially through their sexuality (Erchull, Liss, \& Lichiello, 2013; Erchull \& Liss, 2014). According to Liss and Erchull (2015), self-sexualization is characterized as any action taken by an individual that intentionally highlights his or her sexualized features. In this regard, women who engage in body surveillance, using their body as a tool in order to gain social advantages, do not necessarily experience immediate negative consequences. Yet, given the sexualized culture women live in with ubiquitous and unrealistic beauty expectations, even constant body surveillance can fail to protect women from experiencing body shame. Given that body shame is 
by definition the experience of intense feeling of shame when people realize that their expectations fail to attain an ideal - clearly a negative consequence of self-objectification and body surveillance that has potential to impact mental health over time. Thus, having high body surveillance may lead some women to have more (perceived) control over their life and thus feel less anxious and stressed. Others, who see body surveillance or social comparison based on appearance as a need, are more likely to experience body shame, which is highly correlated with psychological distress such as anxiety and stress (e.g., Monro \& Huon, 2005). These differences in term of perception may, in part, be explained by the role of self-compassion in body image.

\section{Moderations and mediated moderation}

The first two steps of the meditated moderation showed that selfcompassion moderated the effect of body surveillance on depression and happiness separately. Indeed, the relationship between body surveillance and happiness or depression became less stringent for those with high self-compassion. In other words, depression levels of women who have high levels of self-compassion remained low regardless of their engagement in more or less body surveillance. In contrast, women low in self compassion experienced more depression than women high in self-compassion, and depression was heightened when engaging in more relative to less body surveillance. The same effect occurs for subjective happiness with women high in self-compassion reporting greater happiness than women low in self-compassion regardless of whether they were engaging in more or less body surveillance. Yet, for women low in self-compassion engaging in more body surveillance led to less happiness than engaging in less body surveillance. Interestingly, the moderation effect was not significant for body shame, anxiety and stress. This may be due to a lack of power in this study because other scholars have found positive effects of selfcompassion on body shame (Liss \& Erchull, 2015), and the beta reported in the present study is trending toward significance (in Tukeys's terminology: Abelson, 1995). Future research with a larger and perhaps more diverse sample should further investigate this relation to form a more nuanced understanding of the role self-compassion plays in buffering women's feelings of body shame (see for example 
Wollast, Riemer, Gervais, Grigoryan \& Klein, 2019). Indeed, from a purely descriptive standpoint, it confirms our hypotheses regarding the beneficial effects of self-compassion among women.

Most importantly, the last step of the mediated moderation was conclusive. Specifically, we found that for women low in self compassion, body surveillance was negatively associated with happiness and this link was explained by greater feelings of depression. In other words, women who are self-compassionate can protect themselves from depression, and thus feel more satisfied in their lives.

\section{Implications and future directions}

Research examining body image from a self-compassionate perspective is flourishing. The associations found in the present study enrich the current literature and suggest implications for research and practice. Research. The majority of studies focusing on self-objectification have failed to consider factors like self-compassion that may have the power to counteract the detrimental effects of body surveillance. This study overcomes this limitation and addresses several associations between these concepts. Interestingly, we found strong and promising relations between the way women see and treat themselves. In this regard, we invite scholars to step outside of the typical analyses made in the literature and start considering the phenomenon of self-objectification in the light of self-compassion.

For example, there is an active fat acceptance movement (Sturmer, Simon, Loewy \& Jorger, 2003) that encourages larger women to develop feelings of empowerment about their size and leads them to experience many benefits of being fat-accepting, such as self-acceptance, emancipation from dieting, and more time and energy to pursue other interests (Donaghue \& Clemitshaw, 2012). Future research should explore this possibility in greater details to determine if a relationship exists among weight, self-sexualization, body surveillance, happiness and depression.

Practice. Recently, scholars have demonstrated the positive effect of meditation (Albertson et al., 2014) and yoga (Cox et al., 2016, 2017) on body image. In line with these meditation interventions, the results of our study may help women improve their quality of life. Specifically, 
these results suggest that practice professionals such as therapists, counselors, activists, social policy makers, instructors, and organizational administrators should include self-compassion interventions within their training and mentoring efforts. In sum, the findings of the current study may assist in the identification of key factors associated with body image so that practitioners can develop and deliver effective interventions.

\section{Limitations}

Despite the theoretical and practical implications, the current work, like all research, is not without its limitations. In particular, the current study relied upon a cross-sectional research design. While this work reveals that self-compassion plays a role in the relation between body surveillance and feelings of depression and happiness, the nature of the study design fails to determine the direction of this relation. It is possible that women who feel greater body shame engage in more body surveillance, and that self-compassion moderates this link. While this might be an interesting avenue for future research, objectification theory suggests, however, that women engage in body surveillance as a result of cultural pressure stressing the importance of physical appearance management and feel shame as a result of this body surveillance (Fredrickson \& Roberts, 1997).

The current study also relied on a relatively homogeneous sample. Importantly, beauty ideals are shaped by cultural ideals and, as a result, women's level of body surveillance has been found to vary by demographic characteristics such as race, socioeconomic status, and sexual orientation (for a review see Moradi \& Huang, 2008). Because self-objectification operates differently based on intersectional identities, it will be essential for future research to examine the role of selfcompassion in women's diverse experiences of self-objectification (see for example Wollast, Riemer, Gervais et al., 2019). Moreover, the current work focused specifically on women's self-objectification. While our focus on women was intentional because of the pervasive nature of objectification and subsequent self-objectification in women's lives (Fredrickson \& Roberts, 1997), men also engage in body surveillance and experience body shame (see Moradi \& Huang, 2008 for review), suggesting future work should examine the role of self-compassion in 
men's experiences of self-objectification. Although men may engage in body surveillance, the cultural beauty expectations placed on men may be less unforgiving and therefore men may be more self-compassionate regarding their appearance than women (see for example Wollast, Riemer, Sarda \& Klein, 2019). Yet, this is an empirical question, and future research should also examine whether self-compassion plays a similar role in the relation between body surveillance and body shame among men.

\section{Conclusion}

These encouraging results suggest that self-compassion plays a beneficial role by protecting women against the harmful appearancefocused milieu they are living in. Specifically, we revealed that for women high in self-compassion engaging in frequent body surveillance did not necessarily lead to negative consequences. While body surveillance did not generate depression or unhappiness for women high in self-compassion, it is important to point out that body surveillance also did not lead to greater well-being; while body surveillance may not be quite as harmful to women high in self-compassion, women may be happiest if they learn to end habitual body surveillance. Given the culture women are socialized in, ending body surveillance may not only be difficult, but it may also strip women of the possibility to act as their own first surveyor and gain limited feelings of power. These results suggest that interventions focused on self-compassion may at least buffer women against the habitual body surveillance they are socialized to enact. We very much hope scholars and practitioners will pursue the paths opened in this paper.

\section{References}

Abelson, R. P. (1995). Statistics as principled argument. Hillsdale, NJ: Erlbaum.

Albertson, E. R., Neff, K. D. \& Dill-Shackleford, K. E. (2014). Self-compassion and body dissatisfaction in women: A randomized control trial of a brief mediation intervention. Mindfulness, 6, 444- 454 .

Bartels, M. \& Boomsma, D. I. (2009). Born to be happy? The etiology of subjective well-being. Behavior Genetics, 39, 605-615.

Bartky, S. L. (1990). Femininity and domination: Studies in the phenomenology of oppression. New York: Routledge. 
Braun, T. D., Park, C. L. \& Gorin, A. (2016). Self-compassion, body image, and disordered eating: A review of the literature. Body Image, 17, 117-131.

Breines, J. G., Crocker, J. \& Garcia, J. A. (2008). Self-objectification and well-being in women's daily lives. Personality and Social Psychology Bulletin, 34, 583-598.

Brislin, R. W. (1970). Back-translation for cross-cultural research. Journal of Cross-Cultural Psychology, 1, 185-216.

Cox, A. E., Ullrich-French, S., Cole, A. N. \& D'Hondt-Taylor, M. (2016). The role of state mindfulness during yoga in predicting self-objectification and reasons for exercise. Psychology of Sport and Exercise, 22, 321-327.

Cox, A. E., Ullrich-French, S., Howe, H. S. \& Cole, A. N. (2017). A pilot yoga physical education curriculum to promote positive body image. Body Image, 23, 1-8.

Crawford, J. R. \& Henry, J. D. (2003). The Depression Anxiety Stress Scales (DASS): Normative data and latent structure in a large nonclinical sample. British Journal of Clinical Psychology, 42, 111-131.

Daye, C. A., Webb, J. B. \& Jafari, N. (2014). Exploring self-compassion as a refuge against recalling the body-related shaming of caregiver eating messages on dimensions of objectified body consciousness in college women. Body Image, 11, 547-556.

Donaghue, N. \& Clemitshaw, A. (2012). "I'm totally smart and a feminist ... and yet I want to be a waif": Exploring ambivalence towards the thin ideal within the fat acceptance movement. Women's Studies International Forum, 35, 415-425.

Easterlin, R. A. (2003). Explaining happiness. Proceedings of the National Academy of Sciences, 100, 11176-11183.

Erchull, M. J. \& Liss, M. (2014). The object of one's desire: How perceived sexual empowerment through objectification is related to sexual outcomes. Sexuality \& Culture, $18,773-788$.

Erchull, M. J., Liss, M. \& Lichiello, S. (2013). Extending the negative consequences of media internalization and self-objectification to dissociation and self-harm. Sex roles, 69, 583-593.

Fardouly, J., Diedrichs, P. C., Vartanian, L. R. \& Halliwell, E. (2015). Social comparisons on social media: The impact of Facebook on young women's body image concerns and mood. Body Image, 13, 38-45.

Ferreira, C., Pinto-Gouveia, J. \& Duarte, C. (2013). Self-compassion in the face of shame and body image dissatisfaction: Implications for eating disorders. Eating Behaviors, 14, 207-210.

Flynn, M. A., Craig, C. M., Anderson, C. N. \& Holody, K. J. (2016). Objectification in popular music lyrics: An examination of gender and genre differences. Sex Roles, 75, 164-176. Fredrickson, B. L. \& Harrison, K. (2005). Throwing like a girl. Journal of Sport and Social Issues, 29, 79-101.

Fredrickson, B. L. \& Roberts, T. A. (1997). Objectification theory: Toward understanding women's lived experiences and mental health risks. Psychology of Women Quarterly, 21, 173-206. 
Fredrickson, B. L., Roberts, T. A., Noll, S., Quinn, D. M. \& Twenge, J. M. (1998). That swimsuit becomes you: Sex differences in self objectification, restrained eating, and math performance. Journal of Personality and Social Psychology, $75,269-284$.

Germer, C. (2009). The mindful path to self-compassion: Freeing yourself from destructive thoughts and emotions. New York: Guilford Press.

Gervais, S. J., Vescio, T. K. \& Allen, J. (2011). When what you see is what you get: The consequences of the objectifying gaze for women and men. Psychology of Women Quarterly, 35, 5-17.

Gilbert, P. \& Procter, S. (2006). Compassionate mind training for people with high shame and self-criticism: Overview and pilot study of a group therapy approach. Clinical Psychology and Psychotherapy, 13, 353-379.

Hall, C. W., Row, K. A., Wuensch, K. L. \& Godley, K. R. (2013). The role of self-compassion in physical and psychological well-being. The Journal of psychology, 147, 311-323.

Hall, C. W., Row, K., Wuensch, K., Godley, K. \& Edwards, L. (2011). Relationship between self-compassion and emotional intelligence. PsycEXTRA Dataset. https://doi.org/10.1037/e705242011-001

Hatton, E. \& Trautner, M. N. (2011). Equal opportunity objectification? The sexualization of men and women on the cover of Rolling Stone. Sexuality \& Culture, 15, 256-278.

Heffernan, M., Quinn Griffin, M. T., McNulty, S. R. \& Fitzpatrick, J. J. (2010). Selfcompassion and emotional intelligence in nurses. International Journal of Nursing Practice, 16, 366-373.

Hollis-Walker, L. \& Colosimo, K. (2011). Mindfulness, self-compassion, and happiness in non-mediators: A theoretical and empirical examination. Personality and Individual Differences, 50, 222-227.

Hutchinson, M. G. (1985). Transforming body image: Learning to love the body you have. Berkeley, CA: The Crossing Press.

Karsay, K. \& Matthes, J. (2016). Sexually objectifying pop music videos, young women's self-objectification, and selective exposure. Communication Research, $1-23$.

Kelly, A. C., Carter, J. C. \& Borairi, S. (2014). Are improvements in shame and selfcompassion early in eating disorders treatment associated with better patient outcomes? International Journal of Eating Disorders, 47, 54-64.

Kelly, A. C., Vimalakanthan, K. \& Miller, K. E. (2014). Self-compassion moderates the relationship between body mass index and both eating disorder pathology and body image flexibility. Body Image, 11, 446-453.

Kotsou, I. \& Leys, C. (2016). Self-Compassion Scale (SCS): Psychometric properties of the French translation and its relations with psychological wellbeing, affect and depression. PLOS ONE, 11, e0152880.

Kotsou, I. \& Leys, C. (2017). Echelle de bonheur subjectif (SHS): Proprieties psychométriques de la version francaise de l'echelle (SHSF) et ses relations avec le bien-être psychologique, l'affect et la depression. Canadian Journal of Behavioural Science/Revue Canadienne des Sciences du Comportement, 49, 1-6. 
Leys, C., Arnal, C., Wollast, R., Rolin, H., Kotsou, I. \& Fossion, P. (2018). Perspectives on resilience: Trait or aptitude? European Journal of Trauma \& Dissociation, 39, 22-28.

Liss, M. \& Erchull, M. J. (2015). Not hating what you see: Self-compassion may protect against negative mental health variables connected to selfobjectification in college women. Body Image, 14, 5-12.

Lovibond, P. F. \& Lovibond, S. H. (1995). The structure of negative emotional states: Comparison of the Depression Anxiety Stress Scales (DASS) with the Beck Depression and Anxiety Inventories. Behaviour Research and Therapy, 33, 335-343.

Lyubomirsky, S. \& Lepper, H. S. (1999). A measure of subjective happiness: Preliminary reliability and construct validation. Social indicators research, 46, 137-155.

Lyubomirsky, S., King, L. \& Diener, E. (2005). The benefits of frequent positive affect: Does happiness lead to success? Psychological Bulletin, 131, 803-855.

MacBeth, A. \& Gumley, A. (2012). Exploring compassion: A metaanalysis of the association between self-compassion and psychopathology. Clinical Psychology Review, 32, 545-552.

Magnus, C. M., Kowalski, K. C. \& McHugh, T. L. F. (2010). The role of selfcompassion in women's self-determined motives to exercise and exerciserelated outcomes. Self and Identity, 9, 363-382.

Mattei, D. \& Schaefer, C. E. (2004). An investigation of validity of the subjective happiness scale. Psychological Reports, 94, 288-290.

McKinley, N. M. \& Hyde, J. S. (1996). The objectified body consciousness scale development and validation. Psychology of Women Quarterly, 20, 181-215.

Mercurio, A. E. \& Landry, L. J. (2008). Self-objectification and wellbeing: The impact of self-objectification on women's overall sense of self-worth and life satisfaction. Sex Roles, 58, 458-466.

Miner-Rubino, K., Twenge, J. M. \& Fredrickson, B. L. (2002). Trait selfobjectification in women: Affective and personality correlates. Journal of Research in Personality, 36, 147-172.

Monro, F. \& Huon, G. (2005). Media-portrayed idealized images, body shame, and appearance anxiety. International Journal of Eating Disorders, 38, 85-90.

Moradi, B. \& Huang, Y. P. (2008). Objectification theory and psychology of women: A decade of recent advances and future directions. Psychology of Women Quarterly, 32, 377-398.

Morry, M. M. \& Staska, S. L. (2001). Magazine exposure: Internalization, selfobjectification, eating attitudes, and body satisfaction in male and female university students. Canadian Journal of Behavioural Science/Revue Canadienne des Sciences du Comportement, 33, 269-279.

Mosewich, A. D., Kowalski, K. C., Sabiston, C. M., Sedgwick, W. A. \& Tracy, J. L. (2011). Self-compassion: A potential resource for young women athletes. Journal of Sport and Exercise Psychology, 33, 103-123. 
Muehlenkamp, J. J. \& Saris-Baglama, R. N. (2002). Self-objectification and its psychological outcomes for college women. Psychology of Women Quarterly, 26, 371-379.

Muller, D., Judd, C. M. \& Yzerbyt, V. Y. (2005). When moderation is mediated, and mediation is moderated. Journal of personality and social psychology, 89, 852-863.

Neely, M. E., Schallert, D. L., Mohammed, S. S., Roberts, R. M. \& Chen, Y.-J. (2009). Self-kindness when facing stress: The role of self-compassion, goal regulation, and support in college students' wellbeing. Motivation and Emotion, 33, 88-97.

Neff, K. D. (2003). The development and validation of a scale to measure selfcompassion. Self and Identity, 2, 223-250.

Neff, K. D. \& Germer, C. K. (2013). A pilot study and randomized controlled trial of the mindful self-compassion program. Journal of Clinical Psychology, 69, 28-44.

Neff, K. D., Hsieh, Y. P. \& Dejitterat, K. (2005). Self-compassion, achievement goals, and coping with academic failure. Self and Identity, 4, 263-287.

Neff, K. D., Kirkpatrick, K. L. \& Rude, S. S. (2007). Self-compassion and adaptive psychological functioning. Journal of Research in Personality, 41, 139-154.

Neff, K. D. \& McGehee, P. (2010). Self-compassion and psychological resilience among adolescents and young adults. Self and Identity, 9, 225-240.

Noll, S. M. \& Fredrickson, B. L. (1998). A mediational model linking selfobjectification, body shame, and disordered eating. Psychology of Women Quarterly, 22, 623-636.

Olson, K., Kemper, K. J. \& Mahan, J. D. (2015). What factors promote resilience and protect against burnout in first-year pediatric and medicine-pediatric residents? Journal of Evidence-Based Complementary \& Alternative Medicine, 20, 192-198.

Peat, C. M. \& Muehlenkamp, J. J. (2011). Self-objectification, disordered eating, and depression. Psychology of Women Quarterly, 35, 441-450.

Pisitsungkagarn, K., Taephant, N. \& Attasaranya, P. (2014). Body image satisfaction and self-esteem in Thai female adolescents: The moderating role of self-compassion. International Journal of Adolescent Medicine and Health, 26, 333-338.

Podsakoff, P. M., MacKenzie, S. B., Lee, J. Y. \& Podsakoff, N. P. (2003). Common method biases in behavioral research: A critical review of the literature and recommended remedies. Journal of Applied Psychology, 88, 879-903.

Prowse, E., Bore, M. \& Dyer, S. (2013). Eating disorder symptomology, body image, and mindfulness: Findings in a non-clinical sample. Clinical Psychology, $17,77-87$.

Raes, F. (2011). The effect of self-compassion on the development of depression symptoms in a non-clinical sample. Mindfulness, 2, 33-36.

Raes, F., Pommier, E., Neff, K. D. \& Van Gucht, D. (2011). Construction and factorial validation of a short form of the self-compassion scale. Clinical Psychology and Psychotherapy, 18, 250-255. 
Rahimi-Ardabili, H., Reynolds, R., Vartanian, L. R., McLeod, L. V. D. \& Zwar, N. (2017). A Systematic review of the efficacy of interventions that aim to increase self-compassion on nutrition habits, eating behaviours, body weight and body image. Mindfulness, 9, 388-400.

Ramasawmy, S. (2015). Validation of the French Depression Anxiety Stress Scales (DASS-21) and predictors of depression in an adolescent Mauritian population (Doctoral dissertation, Aix-Marseille).

Schimmack, U. (2008). The structure of subjective well-being. In M. Eid \& R. J. Larsen (Eds.), The science of subjective well-being (pp. 97- 123). New York: Guilford Press.

Sturmer, S., Simon, B., Loewy, M. \& Jorger, H. (2003). The dualpathway model of social movement participation: The case of the fat acceptance movement. Social Psychology Quarterly, 66, 71-82.

Swami, V., Tran, U. S., Stieger, S. \& Voracek, M. (2015). Associations between women's body image and happiness: Results of the YouBeauty.com Body Image Survey (YBIS). Journal of Happiness Studies, 16, 705-718.

Szymanski, D. M. \& Henning, S. L. (2007). The role of self-objectification in women's depression: A test of objectification theory. Sex Roles, 56, 45-53.

Szymanski, D. M., Moffitt, L. B. \& Carr, E. R. (2011). Sexual objectification of women: Advances to theory and research. The Counseling Psychologist, 39, 6-38.

Thompson, B. L. \& Waltz, J. (2008). Self-compassion and PTSD symptom severity. Journal of Traumatic Stress, 21, 556-558.

Tylka, T. L. \& Hill, M. S. (2004). Objectification theory as it relates to disordered eating among college women. Sex Roles, 51, 719-730.

Vandenbosch, L. \& Eggermont, S. (2012). Sexually explicit websites and sexual initiation: Reciprocal relationships and the moderating role of pubertal status. Journal of Research on Adolescence, 23, 621-634.

Vandenbosch, L. \& Eggermont, S. (2013). Sexualization of adolescent boys: Media exposure and boys' internalization of appearance ideals, self-objectification, and body surveillance. Men and Masculinities, 16, 283-306.

Vandenbosch, L. \& Eggermont, S. (2014). The three-step process of selfobjectification: Potential implications for adolescents' body consciousness during sexual activity. Body Image, 11, 77-80.

Veenhoven, R. (2010). How universal is happiness? In D. Daniel Kahneman \& J. Helliwell (Eds.), International differences in wellbeing. New York, NY: Oxford University Press.

Wallace, S. H. \& Brislin, R. W. (1973). Evaluation language translations: Experiments on three assessment methods. Journal of Applied Psychology, 57, 328-334.

Ward, L. M., Seabrook, R. C., Manago, A. \& Reed, L. (2016). Contributions of diverse media to self-sexualization among undergraduate women and men. Sex Roles, 74, 12-23. 
Wasylkiw, L., MacKinnon, A. L. \& MacLellan, A. M. (2012). Exploring the link between self-compassion and body image in university women. Body Image, 9, 236-245.

Webb, J. B. \& Forman, M. J. (2013). Evaluating the indirect effect of selfcompassion on binge eating severity through cognitive-affective self-regulatory pathways. Eating Behaviors, 14, 224-228.

Werner, K. H., Jazaieri, H., Goldin, P. R., Ziv, M., Heimberg, R. G. \& Gross, J. J. (2012). Self-compassion and social anxiety disorder. Anxiety Stress and Coping, 25, 543-558.

Wollast, R., Coertjens, L., Bernard, P. \& Klein, O. (2019). Is the relation between sexism and body image culture-dependent? A Comparison between Belgium and Thailand. Manuscript under revision.

Wollast, R., Puvia, E., Bernard, P., Tevichapong, P. \& Klein, O. (2018). How sexual objectification generates dehumanization in Western and Eastern cultures: A comparison between Belgium and Thailand. Swiss Journal of Psychology, 77, 69-82.

Wollast, R., Riemer, A., Gervais, S., Grigoryan, L. \& Klein, O. (2019). How selfcompassion moderates the effect of body surveillance on body shame for women from America, Belgium, Russia, and Thailand. Manuscript under revision.

Wollast, R., Riemer, A., Sarda, E. \& Klein, O. (2019). How self-compassion moderates the relation between body surveillance on body shame among men and women. Manuscript under revision. 